\title{
Associação da vitamina B na esquizofrenia
}

A esquizofrenia é um distúrbio neuropsiquiátrico grave que não só causa uma grande carga de doenças, mas também desafia nossa compreensão de como a mente e o cérebro funcionam. A esquizofrenia afeta em torno de $1 \%$ da população mundial, somente no Brasil mais de 2 milhões de pessoas sofrem com esse distúrbio e segundo a Associação Brasileira de Psiquiatria, a doença é predominante no sexo masculino e normalmente não é diagnosticada no início. O diagnóstico é baseado em uma constelação de sintomas clínicos e não em um pato mecanismo comum. A vitaminas são compostos orgânicos, necessários em pequenas quantidades para o metabolismo normal e que não podem ser fabricadas pelas células do corpo. As hidrossolúveis são absorvidas pelo intestino e transportadas pelo sistema circulatório até os tecidos em que serão utilizadas, não são armazenadas e são facilmente excretadas pelos rins, por isso devem ser ingeridas diariamente, neste grupo estão as vitaminas B. O complexo B pode ser usado como coenzimas no catabolismo de macronutrientes para produzir energia para 0 corpo humano. Pesquisas demonstram que tratamento auxiliar com vitaminas B com uma alta dose - incluindo B6, B8 e B12 - pode significativamente reduzi sintomas de tratamentos mais do que padrão da esquizofrenia apenas. Uma pesquisa bibliográfica foi feita utilizando as bases de dados Google acadêmico, Scielo, Pubmed e Lilacs, com esquizofrenia, complexo B, vitamina B e espectro de esquizofrenia como descritores. 21 artigos foram incluídos que atenderam os critérios de inclusão. Em conclusão, a administração de vitaminas B6, B9 E B12 tiveram resultados positivos em relação a memória e a sintomas negativos de paciente esquizofrênicos, também é necessário o desenvolvimento de um novo modelo animal, pois a utilização do atual para o estudo da esquizofrenia ainda não é eficiente.

Palavras-chave: Esquizofrenia; Complexo B; Vitamina B; Espectro de Esquizofrenia.

\section{Association of vitamin B in schizophrenia}

Schizophrenia is a serious neuropsychiatric disorder that not only causes a great deal of disease, but also challenges our understanding of how the mind and brain work. Schizophrenia affects around 1\% of the world population, just in Brazil more than 2 million people suffer from this disorder and according to the Brazilian Psychiatric Association, the disease is prevalent in males and is usually not diagnosed at the beginning. The diagnosis is based on a constellation of clinical symptoms and not on a common pathomechanism. Vitamins are organic compounds, needed in small amounts for normal metabolism and that cannot be manufactured by the cells of the body. Water-soluble substances are absorbed by the intestine and transported through the circulatory system to the tissues in which they will be used, they are not stored and are easily excreted by the kidneys, so they must be ingested daily, in this group are vitamins B. The B complex can be used as coenzymes in macronutrient catabolism to produce energy for the human body. Research shows that ancillary treatment with $B$ vitamins at a high dose - including B6, B8 and B12 - can significantly reduce symptoms of treatments more than standard schizophrenia alone. A bibliographic search was made using the Google academic databases, Scielo, Pubmed and Lilacs, with schizophrenia, B complex, vitamin B and schizophrenia spectrum as descriptors. 21 articles were included that met the inclusion criteria. In conclusion, the administration of vitamins B6, B9 and B12 had positive results in relation to memory and negative symptoms of schizophrenic patients, it is also necessary to develop a new animal model, since the use of the current one for the study of schizophrenia has not yet it's efficient.

Keywords: Schizophrenia; Complex B; Vitamin B; Schizophrenia Spectrum.

\section{Topic: Neurologia e Neurociências}

Reviewed anonymously in the process of blind peer
Received: $17 / 10 / 2020$

Approved: 19/01/2021
Juliana Veras Soares (iD

Centro Universitário Christus, Brasil

http://lattes.cnpq.br/5592351221105465

http://orcid.org/0000-0001-9785-0682

verasbiomed@gmail.com

Melissa Figueiredo Capelo (iD)

Centro Universitário Christus, Brasil

http://lattes.cnpq.br/0213224523703282

http://orcid.org/0000-0002-9216-519X

melissacapelo012@gmail.com

Ana Beatriz Aguiar Sanford (iD)

Centro Universitário Christus, Brasil

http://lattes.cnpq.br/8849440063851596

http://orcid.org/0000-0001-7496-4572

anabeatrizsanford@gmail.com

\author{
Dara da Silva Mesquita (iD \\ Universidade Federal do Ceará, Brasil \\ http://lattes.cnpq.br/6780805732812263 \\ http://orcid.org/0000-0002-8186-8375 \\ daramesq@gmail.com \\ José Eduardo Ribeiro Honório Júnior \\ Centro Universitário Christus, Brasil \\ http://lattes.cnpq.br/4272610021115688 \\ http://orcid.org/0000-0001-8320-8525 \\ eduribiologo@yahoo.com.br
}

Referencing this:

SOARES, J. V.; CAPELO, M. F.; SANFORD, A. B. A.; MESQUITA, D. S.; HONÓRIO JÚNIOR, J. E. R.. Associação da vitamina $B$ na esquizofrenia. Scire Salutis, v.11, n.1, p.76-83, 2021. DOI:

http://doi.org/10.6008/CBPC2236-9600.2021.001.0009

DOI: 10.6008/CBPC2236-9600.2021.001.0009 


\section{INTRODUÇÃO}

De acordo com a Organização Pan-Americana da Saúde, 23 milhões de pessoas no mundo são portadoras de esquizofrenia. Somente no Brasil, dois milhões de brasileiros apresentam o distúrbio. A esquizofrenia é um distúrbio mental crônico e grave, com psicopatologias que afetam a maneira como a pessoa pensa, sente e se comporta (CAO et al., 2017). Isso pode levar a mudanças de comportamento, apatia emocional, confusão e dificuldade em lidar com as pessoas. Esse distúrbio afeta principalmente jovens que estão começando a atingir a idade adulta ou atingiram o pico de produção (COSTA et al., 2013).

Devido à intensidade dos sintomas, um grande retraimento social é descrito nesses pacientes; por conta do surgimento de pesquisas foi possível perceber que é difícil para a pessoa afetada com esse transtorno aceitarem que tem essa psicose, pois haverá sentimentos paralelos gerados pelos sintomas psicóticos na vida do paciente (COSTA et al., 2013). Com suas alucinações, delírios, distúrbios de pensamento e déficits cognitivos, a esquizofrenia afeta os processos humanos mais básicos de percepção, emoção e julgamento (ROSS et al., 2006). Em relação às mudanças neuropsicológicas, a esquizofrenia descreve defeitos seletivos na memória da linguagem e velocidade de processamento (PIÑóN et al., 2018).

A esquizofrenia também é frequentemente descrita como uma doença heterogênea porque muitos sintomas diferentes são vistos e pode haver muitas causas subjacentes desconhecidas (ROSS et al., 2006). Quando os sintomas se desenvolvem, após a puberdade, podem ser classificados em positivo caracterizado por alucinações e delírios; em negativo, isolamento social, anedonia e apatia; e déficit cognitivo. Atualmente o tratamento da esquizofrenia é feito pela combinação de drogas antipsicóticas e psicoterapia, para ter maior eficiência no tratamento, mas mesmo assim não possui grande influência nos sintomas negativos, tendo efeitos satisfatórios apenas nos positivos (ELLENBROEK, 2017).

O diagnóstico é baseado no enquadramento de sintomas clínicos, não em mecanismos patogenéticos comuns, como no acidente vascular cerebral isquêmico ou ataque cardíaco. Os dois sistemas de diagnóstico mais amplamente usados, a Classificação Estatística Internacional de Doenças e Problemas Relacionados à Saúde (CID-10) e o 5o Manual Diagnóstico e Estatístico de Transtornos Mentais, Associação Psiquiátrica Americana (DSM-V) fornecem catálogos de sintomas. E um certo número de grupos deve existir dentro de um certo período de tempo para o diagnóstico. De acordo com o DSM-V, pacientes que apresentam alucinações e alucinações podem ser diagnosticados com esquizofrenia, assim como pacientes com confusão na fala e sintomas negativos (NICKL-JOCKSCHAT et al., 2016). Um dos tratamentos utilizados atualmente é o uso de antioxidantes, como as vitaminas, que estabilizam o efeito do estresse oxidativo, já que pesquisas mostram o envolvimento desse desequilíbrio oxidativo no estabelecimento da esquizofrenia (QUEIROZ et al., 2012).

As vitaminas são compostos orgânicos que requerem uma pequena quantidade para serem metabolizados normalmente e não podem ser produzidos pelas células humanas. Eles são essenciais porque estão envolvidos em vários processos e mecanismos importantes, como: coenzimas e antioxidantes (IMBRAIM et al., 2016). Elas são divididas em dois grupos, hidrossolúveis e lipossolúveis, sendo classificadas 
com base nas características físico-químicas e pela ação biológica. Dentro do grupo das hidrossolúveis está o complexo B (Figura 1), formado por Tiamina (B1), Riboflavina (B2), Niacina (B3), Ácido Pantoténico (B5), Piridoxina (B6), Biotina (B7), Ácido Fólico (B9), Cobalamina (B12), Ácido Pangâmico (B15) e Paba (BX).

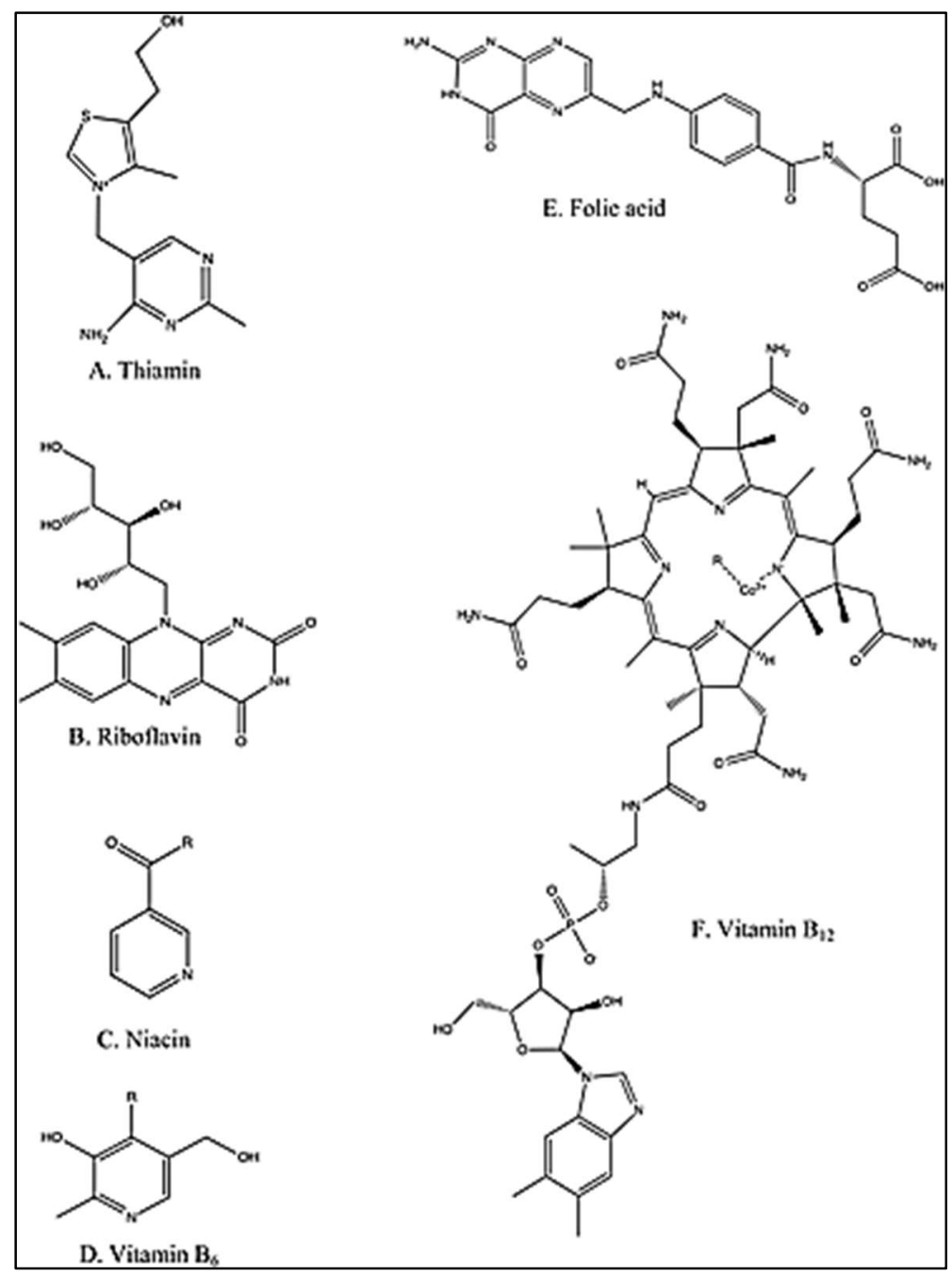

Figura 1: Estruturas químicas (A) tiamina, (B) riboflavina, (C) niacina, (D) vitamina B6, (E) ácido fólico e (F) vitamina B12.

A maioria das vitaminas B podem ser usadas como coenzimas no catabolismo de macronutrientes para produzir energia para o corpo humano. Este grupo de vitaminas é necessário para o corpo humano em pequenas quantidades e deve ser obtido dos alimentos, pois não são sintetizadas pelo organismo (RUBERT et al., 2017). Relacionando a vitamina B com a esquizofrenia, a vitamina B é usada na síntese e metabolismo de neurotransmissores e no desenvolvimento e preservação da memória. As vitaminas B1, B3, B5, B6 e B12 irão convergir na memória através de diferentes mecanismos de ação. Por exemplo, uma certa parte da cianocobalamina (Figura 1) desempenha um papel muito importante na manutenção do metabolismo dos ácidos graxos necessário para a mielina (QUEIROZ et al., 2012). Assim, a partir do que foi exposto acima, temos como objetivo analisar, por meio de uma revisão literária, a eficácia da vitamina B na esquizofrenia e como ela pode atuar no tratamento. 


\section{METODOLOGIA}

Foi realizada uma pesquisa bibliográfica utilizando bases de dados eletrônicas (Google acadêmico, Scielo, Pubmed e Lilacs) para artigos, publicados em inglês, português e espanhol, entre 2005 e 2020. Os descritores utilizados foram esquizofrenia, espectro de esquizofrenia, complexo B e vitamina B.

\section{RESULTADOS E DISCUSSÃO}

Primeiramente, a busca inicial rendeu um total de 543 artigos, porém com os critérios de inclusão, somente 200 se tornaram elegíveis. Após a análise dos resumos dos artigos e comparando as informações com o tema deste artigo, 24 trabalhos foram escolhidos para a leitura de texto completo. Apenas 20 artigos foram incluídos nesta revisão.

Tabela 1: Relação dos resultados obtidos dos principais artigos.

\begin{tabular}{|c|c|c|}
\hline AUTOR/ANO & $\begin{array}{l}\text { TIPO DE } \\
\text { ESTUDO }\end{array}$ & RESULTADO \\
\hline $\begin{array}{l}\text { Queiroz et al., } \\
2012\end{array}$ & Clínico & $\begin{array}{l}\text { Evidenciou que os mecanismos oxidativos na fisiopatologia da esquizofrenia causa a produção de } \\
\text { radicais livres, prejudicando a memória e os sintomas do paciente, e indicou que o uso das } \\
\text { vitaminas B como antioxidante é muito importante para proteger o tecido cerebral. }\end{array}$ \\
\hline $\begin{array}{l}\text { Mitchell et al., } \\
2014\end{array}$ & Clínico & $\begin{array}{l}\text { Relacionou fatores genéticos com a esquizofrenia. Os resultados mostraram a possível associação } \\
\text { da patologia com o polimorfismo genético da enzima metilenotetrahidrofolato redutase } \\
\text { (MTHFR), necessária para a metabolização da homocisteína (aminoácido que, em excesso, torna- } \\
\text { se um fator de risco para o desenvolvimento da esquizofrenia). }\end{array}$ \\
\hline $\begin{array}{l}\text { Nishi et al., } \\
2014\end{array}$ & Clínico & $\begin{array}{l}\text { O estudo evidenciou que em pacientes esquizofrênicos os níveis de homocisteína no plasma é } \\
\text { maior do que do grupo controle, levando em conta os genótipos da MTHFR, a idade e o gênero } \\
\text { das pessoas envolvidas na pesquisa. }\end{array}$ \\
\hline $\begin{array}{l}\text { Brown et al., } \\
2016\end{array}$ & Clínico & $\begin{array}{l}\text { Relatou que o tratamento, de } 140 \text { pacientes com esquizofrenia, foi mais eficaz no grupo teste que } \\
\text { foi tratado com ácido fólico e vitamina B12, em conjunto com o antipsicótico já usado pelo } \\
\text { paciente. Mostrando uma melhora nos sintomas negativos }\end{array}$ \\
\hline $\begin{array}{l}\text { Cao et al., } \\
2017\end{array}$ & Clínico & $\begin{array}{l}\text { O artigo analisou a relação entre seis tipos de vitamina B e a esquizofrenia. Dessas vitaminas, } \\
\text { baixos níveis de B6 e altos níveis de B3 (após ajuste das variáveis) mostraram-se associadas com a } \\
\text { patologia. }\end{array}$ \\
\hline $\begin{array}{l}\text { Ellenbroek, } \\
2017\end{array}$ & $\begin{array}{l}\text { Pré- } \\
\text { clínico }\end{array}$ & $\begin{array}{l}\text { Indicou que o modelo animal atual não é adequado para o estudo por completo da esquizofrenia, } \\
\text { apontando que os modelos focados na etiologia da esquizofrenia são mais baseados em } \\
\text { hipóteses do que em fatos. Pesquisadores começaram a desenvolver alternativas, como a } \\
\text { administração aguda ou subcrônica de drogas psicotomiméticas, como anfetaminas ou } \\
\text { fenciclidina. }\end{array}$ \\
\hline $\begin{array}{l}\text { Firth et al., } \\
2017\end{array}$ & Clínico & $\begin{array}{l}\text { Não foram encontrados resultados significativos da ação individual das vitaminas B6, B9 e B12. } \\
\text { Somente quando administradas em conjunto, em grandes dosagens e nos primeiros anos da } \\
\text { doença, foi possível observar melhora nos sintomas psiquiátricos da esquizofrenia. }\end{array}$ \\
\hline $\begin{array}{l}\text { Martínez, } \\
2017\end{array}$ & Clínico & $\begin{array}{l}\text { Expôs a ligação entre as vitaminas B6, B9 e B12 com a esquizofrenia. A deficiência delas permite a } \\
\text { alta concentração de homocisteína em esquizofrênicos, podendo ser uma das causas para o } \\
\text { desenvolvimento da patologia. } \\
\text { Além disso, pacientes com a doença apresentaram deficiência de vitamina B9 no organismo em } \\
\text { comparação com o grupo controle. }\end{array}$ \\
\hline $\begin{array}{l}\text { Mitra et al., } \\
2017\end{array}$ & Clínico & $\begin{array}{l}\text { Mostrou que pesquisas que fizeram a suplementação diária de } 2 \mathrm{mg} \text { de B9 e } 400 \mu \mathrm{g} \text { de B12 no } \\
\text { tratamento de esquizofrênicos foi associada com a melhora de sintomas negativos. Enquanto a } \\
\text { suplementação somente de } 2 \mathrm{mg} \text { de B9 não resultou melhora no caso do paciente. }\end{array}$ \\
\hline
\end{tabular}

Estudos mostraram que pacientes com esquizofrenia possuem uma tendência de desenvolver deficiência de vitaminas (BROWN et al., 2016), essas pesquisas realizadas na Espanha e Austrália constataram que pacientes com psicose comem menos de quatro porções de frutas e vegetais recomendados pela Organização Mundial da Saúde (OMS) (BALANZÁ et al., 2017). Então foi sugerido um tratamento com a suplementação de vitaminas, adjunto do convencional, pois existem mecanismos biológicos que podem exercer efeitos positivos por meio desses nutrientes (FIRTH et al., 2017). 
Dessa forma, foi evidenciado que a vitamina B é crucial no metabolismo celular, incluindo reações de transmetilação e oxidação (BROWN et al., 2016), assim indicadores mostraram que a redução de vitaminas D e B no organismo estão associadas a gravidade da esquizofrenia, principalmente em relação aos sintomas negativos (FIRTH et al., 2017). Seguindo essa linha de raciocínio, outras pesquisas foram feitas e concluíram que pacientes com esquizofrenia têm deficiência de vitamina B9, porém, quando essa deficiência é materna tem relação com risco de desenvolver a esquizofrenia, e quando essa deficiência ocorre em pacientes já esquizofrénicos está relacionada a uma maior severidade dos sintomas negativos (BALANZÁ et al., 2017), deterioração do hipocampo e deficiências cognitivas (FIRTH et al., 2017).

Para provar essa afirmação os seguintes estudos foram feitos, Goff et al. (2004), demonstraram a baixa concentração da vitamina B9 em 91 pacientes esquizofrênicos, e depois foi constatado uma relação inversa entre a vitamina B9 no sangue com os sintomas negativos (BROWN et al., 2016). Roffman et al. (2013), trataram esquizofrênicos em antipsicóticos com vitamina B9, B12 ou placebo, e foi concluído que o grupo recebendo tratamento teve melhora na severidade dos sintomas negativos (BROWN et al., 2016). Firth et al. (2017), suplementaram pacientes esquizofrénicos com vitaminas B9, B6 e B12, e chegaram à conclusão que essa suplementação foi definitivamente mais eficiente que o placebo, porém a eficiência foi somente considerável quando foram administradas em doses maiores e em combinação com outras vitaminas, estando nos estados iniciais da esquizofrenia (BALANZÁ et al., 2017).

Como referido anteriormente, a deficiência de vitaminas em pacientes esquizofrênicos é muito prejudicial, por estarem associadas a anormalidades neurológicas, podendo estar ligada ao estresse oxidativo na fisiopatologia da esquizofrenia. Esse estresse ocorre quando existe um desequilíbrio entre os processos antioxidantes e pró-oxidantes, gerando um aumento na formação de radicais livres, esse processo patológico deixa o SNC muito vulnerável, por causa da sua alta taxa de consumo de oxigênio, dos elevados níveis de lipídios poli-insaturados capazes de sofrer peroxidação lipídica, e da auto-oxidação de alguns neurotransmissores (QUEIROZ et al., 2012)

Estudos já mostram que o aumento do estresse oxidativo e a diminuição da atividade antioxidante é mais observada em estados crônicos da esquizofrenia do que na fase aguda (MITRA et al., 2017) e que a falta da vitamina B6 pode resultar no aumento de radicais livre e de peróxido de hidrogênio, o que agravaria os danos aos neurônios do hipocampo e se envolveria no comprometimento cognitivo desse distúrbio (CAO et al., 2017).

A esquizofrenia além de apresentar maior severidade de sintomas diante do déficit de vitamina $B$, apresenta também deficiência de memória, por causa de sua função cognitiva afetada pelos radicais livres que danificam os neurônios, o sistema límbico e o hipocampo. Assim, pesquisas apontam a importância da suplementação de vitaminas B juntamente com o os antipsicóticos usados no tratamento desse distúrbio, pois a vitamina B tem a função de proteger o tecido cerebral por meio da decomposição dos radicais livres em excesso, tendo ação preventiva e antioxidante (QUEIROZ et al., 2012) e sendo uma forma de tratamento segura e barata para os pacientes (MITRA et al., 2017).

Em um estudo realizado por Cao et al. (2017), verificou-se a relação entre a esquizofrenia e seis tipos 
de vitamina B, sendo elas as vitaminas B2, B3, B6, B7, B9 e B12. Nele, observou-se que baixos níveis de piridoxina (a forma alcoólica da vitamina B6) permite o aumento de radicais livres e de peróxido de hidrogênio, que acarretam em danos a neurônios do hipocampo, resultando em comprometimento cognitivo característico da esquizofrenia. Entretanto, uma análise do efeito da vitamina B6 em pacientes com esquizofrenia, no intuito de observar sua ação nos sintomas psiquiátricos da patologia, mostrou que o uso desta vitamina sozinha não atingiu significância estatística, somente quando combinada com outras vitaminas do complexo B era possível reduzir esses sintomas (FIRTH et al., 2017). Outra forma da vitamina B6, a piridoxamina, não se mostrou relacionada com a esquizofrenia (CAO et al., 2017).

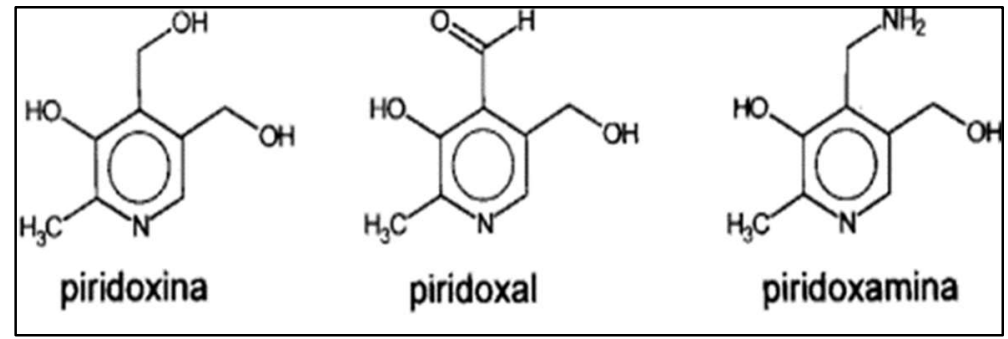

Figura 2: Formas da vitamina B6 Mais comuns encontradas.

Nesse mesmo estudo, a deficiência de ácido fólico (ou vitamina B9) demonstrou-se relacionada com o desenvolvimento de doenças neurais, transtornos mentais e demência (CAO, B. et al., 2017). Entretanto, outras pesquisas mostraram que tratamentos utilizando suplementação de vitamina B9 não apresentou resultados efetivos na diminuição dos sintomas da esquizofrenia; somente quando relacionado aos fatores genéticos dos pacientes envolvidos no estudo, o ácido fólico mostrou-se satisfatório na redução dos sintomas (FIRTH et al., 2017).

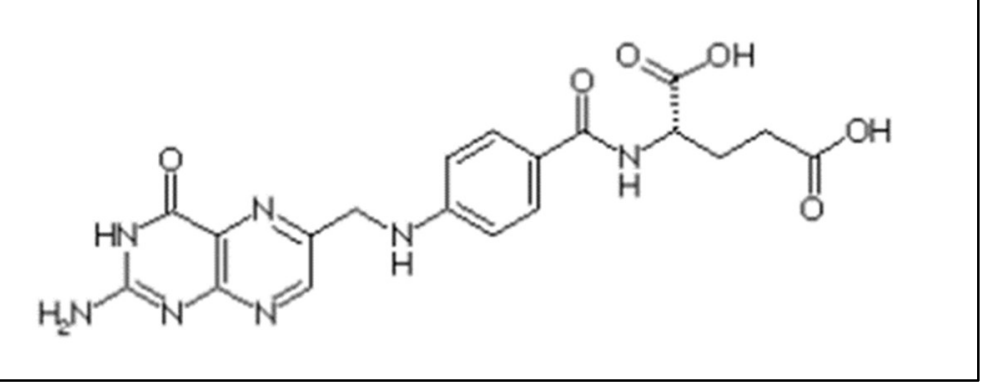

Figura 3: Estrutura química do ácido fólico.

A vitamina B12, também chamada de cianocobalamina, atua no Sistema Nervoso Central (SNC) no metabolismo de ácidos graxo que compõem a bainha de mielina dos neurônios, sendo necessários para a manutenção desta (QUEIROZ et al., 2012). Desse modo, mudanças nos níveis desta vitamina no SNC estão relacionadas com o desenvolvimento de sintomas neuronais (CAO et al., 2017), como os tremores visíveis em pacientes com esquizofrenia (MITCHELL et al., 2014).

As vitaminas B6, B9 e B12 participam do metabolismo da homocisteína: um aminoácido que em excesso leva a hiper-homocisteinemia, fator de risco para doenças cardíacas, problemas cognitivos e transtornos de humor (MARTíNEZ, 2017). A homocisteína $\left(\mathrm{C}_{4} \mathrm{H}_{9} \mathrm{NO}_{2} \mathrm{~S}\right)$ faz parte do ciclo da metionina, onde é sintetizada pela desmetilação da metionina $\left(\mathrm{C}_{5} \mathrm{H}_{11} \mathrm{NO}_{2} \mathrm{~S}\right)$ e deve ser metabolizada por meio uma das duas 
vias: pela transfuração (mediada pela vitamina B6) ou pela remetilação (dependente das vitaminas B9 e B12) (KENNEDY, 2016). Na primeira via citada, a vitamina B6 atua como um cofator para a conversão da homocisteína em cistationina. Já na segunda via, o ácido fólico e a cianocobalamina, que fazem parte do ciclo da síntese de metionina, vão se ligar a enzima metilenotetrahidrofolato redutase (MTHFR) e metabolizar a homocisteína em metionina (MITCHELL et al., 2014).

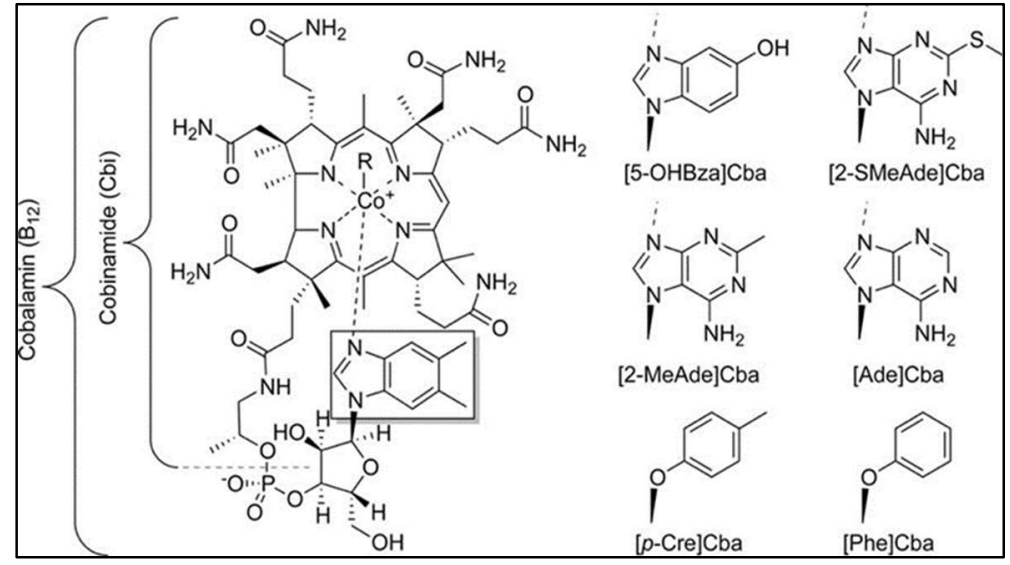

Figura 4: Exemplos representativos de diversidade estrutural corrinoide.

Estudos realizados por Nishi et al. (2014), associou os altos níveis de homocisteína no sangue e o polimorfismo da MTHFR como possíveis fatores de risco para a esquizofrenia. Nos testes de caso-controle realizados, considerando o polimorfismo genético para a enzima MTHFR, a idade e o gênero dos participantes, foram possíveis observar que os níveis de homocisteína no sangue de pacientes esquizofrênicos eram estatisticamente mais altos do que no grupo controle (NISHI et al., 2014). Com esses níveis elevados, a homocisteína é capaz de inibir a reação de metilação, aumentar o estresse oxidativo, e causar danos ao DNA humano, levando a hipótese de ser uma das causas dos problemas cognitivos observado na patologia em questão (KENNEDY, 2016). Entretanto, ainda é necessário a realização de mais estudos sobre a relação do polimorfismo da metilenotetrahidrofolato redutase com a esquizofrenia, uma vez que outra análise dessa relação não mostrou nenhuma ligação significante entre eles (MITCHELL et al., 2014).

Outra vitamina que pode ser associada com a esquizofrenia é a vitamina B3 (niacina ou nicotinamida). Em excesso, a nicotinamida consegue aumentar os níveis de serotonina e histamina no plasma, resultando em um alto metabolismo dessas monoaminas pelo SNC (TIAN et al., 2013). Após ajustar as variáveis, observou-se alta quantidade dessa vitamina em pacientes com esquizofrenia, reforçando a associação desta patologia com as monoaminas (CAO et al., 2017). Um estudo de caso-controle com as vitaminas B2 e B7 não apresentou nenhuma diferença entre pacientes da pesquisa (CAO et al., 2017). Ressalta-se que não foram encontrados artigos sobre a relação das vitaminas B1 e B5 com a esquizofrenia.

\section{CONCLUSÕES}

Por fim, concluímos que a administração de vitaminas B6, B9 e B12 tiveram resultados positivos em relação a memória e a sintomas negativos de pacientes esquizofrênicos, como também o déficit dessas vitaminas foram associadas ao risco do desenvolvimento da esquizofrenia. Contudo, mais estudos a respeito 
devem ser feitos, pois é necessário o desenvolvimento de um novo modelo animal, pois a utilização do modelo atual para o estudo da esquizofrenia ainda não é muito eficiente, por causa da necessidade da replicação da sintomatologia da esquizofrenia em animais, sendo que é uma doença diagnosticada apenas por entrevista psiquiátrica e por seus sintomas ocorrem em várias outras doenças, sendo muito complicado sua confirmação.

\section{REFERÊNCIAS}

BALANZÁ-MARTÍNEZ, V.. Nutritional supplements in psychotic disorders. Actas Espanolas de Psiquiatria, v.45, 2017.

BROWN, H. E.; ROFFMAN, J. L.. Emerging treatments in schizophrenia: Highlights from Recent Supplementation and Prevention Trials. Harv Rev. Psychiatry, v.24, n.2, p.e1-e7, 2016. DOI: http://doi.org/10.1097/HRP.0000000000000101

CAO, B.; SUN, X. Y.; ZHANG, C. B.; YAN, J. J.; ZHAO, Q. Q.; YANG, S. Y.; YAN, L. L.; HUANG, N. H.; ZENG, J.; LIAO, J. Y.; WANG, J. Y.. Association between $B$ vitamins and schizophrenia: a population-based case-control study. Psychiatry Research, v.259, p.501-505, 2017.

COSTA, J. E.; TRAVASSOS, L. C.. Identificação neurocientífica: uma relação entre stress oxidativo e Esquizofrenia. Acervo da Iniciação Científica, n.1, 2013.

ELLENBROEK, B. A.. Schizophrenia. Reference Module in Neuroscience and Biobehavioral Psychology. 2017.

FIRTH, J.; STUBBS, B.; SARRIS, J.; ROSENBAUM, S.; TEASDALE, S.; BERK, M.; YUNG, A. R.. The effects of vitamin and mineral supplementation on symptoms of schizophrenia: a systematic review and meta-analysis. Psychol Med., v.41, n.9, p.1515-1527, 2017.

IMBRAIM, A. C. D. F.; PEREIRA, I. M. C.; PINTO, T. C. F.; PAIVA, A. M. R.. Deficiência de Vitamina de Esquizofrenia. Revista Uningá Review, v.27, n.1, 2016.

KENNEDY, D. O. B.. Vitamins and the Brain: Mechanisms, Dose and Efficacy: A Review. Nutrients, v.8, n.68, p.1-29, 2016.

MARTÍNEZ, V. B.. Nutritional supplements in psychotic disorders. Actas Españolas de Psiquiatría, v.45, p.16-25, 2017.

MITCHELL, E. S.; CONUS, N.; KAPUT, J.. B vitamin polymorphismis and behavior: evidence of associations with neurodevelopment, depression, schizophrenia, bipolar disorder and cognitive decline. Neuroscience and Biobehavioral Review, v.47, p.307-320, 2014.

MITRA, S.; NATARAJAN, R.; ZIEDONIS, D.; FAN, X.. Antioxidant and anti-inflammatory nutrient status, supplementation, and mechanisms in patients with schizophrenia. Progress in Neuropsychopharmacology and Biological Psychiatry, v.78, p.1-11, 2017.
NICKL-JOCKSCHAT, T.; ABEL, T.. Historical and Clinical Overview. In: The Neurobiology of Schizophrenia. 2016. p.313.

NISHI, A.; NUMATA, S.; TAJIMA, A.; KINOSHITA, M.; KIKUCHI, K.; SHIMODERA, S.; TOMOTAKE, M.; OHI, K.; HASHIMOTO, R.; IMOTO, I.; TAKEDA, M.; OHMORI, T.. Meta-analyses of blood homocysteine levels for gender and genetic association studies of the MTHFR C677T polymorphism in schizophrenia. Schizophrenia Bulletin, v.40, n.5, p.11541163, 2014.

PIÑóN, A.; ÁLVAREZ, M.; TORRES, T.; VÁZQUEZ, P.; OTERO, F.. Neuropsychological profile of patients diagnosed with schizophrenia spectrum disorder. Revista de Discapacidad, Clínica y Neurociencias, p.1-14, 2018.

QUEIROZ, A.; OLIVEIRA G.; FREITAS, R.. Associação de antipsicóticos e vitaminas (ácido ascórbico, alfa-tocoferol e complexo b) e seu papel na memória de portadores de esquizofrenia. Revista Eletrônica de Farmácia, v.9, n.2, p.1515, 2012.

ROSS, C. A.; MARGOLIS, R. L.; READING, S. A.; PLETNIKOV, M.; COYLE, J. T.. Neurobiology of schizophrenia. Neuron, v.52, n.1, p.139-153, 2006.

RUBERT, A.; ENGEL, B.; ROHLFES, A. L. B.; MARQUARDT, L.; BACCAR, N. M.. Vitaminas do complexo B: uma breve revisão. Revista Jovens Pesquisadores, v.7, n.1, p.30-45, 2017

TIAN, Y. J.; LI, D.; MA, Q.; GU, X. Y.; GUO, M.; LUN, Y. Z.; SUN, W. P.; WANG, X. Y.; CAO, Y.; ZHOU, S. S.. Excess nicotinamide increases plasma serotonin and histamine levels. Acta Physiologica Sinica, v.65, n.1, p.33-38, 2013.

ROFFMAN, J. L.; LAMBERTI, J. S.; ACHTYES, E.; MACKLIN, E. A.; GALENDEZ, G. C.; RAEKE, L. H.; SILVERSTEIN, N. J.; SMOLLER, J. W.; HILL, M.; GOF, D. C.. Randomized multicenter investigation of folate plus vitamin B12 supplementation in schizophrenia. JAMA Psychiatry, v.70, p.481-489, 2013. DOI: http://doi.org/10.1001/jamapsychiatry.2013.900

GOFF, D. C.; BOTTIGLIERI, T.; ARNING, E.; SHIH, V.; FREUDENREICH, O.; EVINS, A. E.; HENDERSON, D. C.; BAER, L.; COYLE, J.. Folate, homocysteine, and negative symptoms in schizophrenia. Am J Psychiatry, v.161, p.1705-1708, 2004. DOI: http://doi.org/10.1176/appi.ajp.161.9.1705

A CBPC - Companhia Brasileira de Produção Científica (CNPJ: 11.221.422/0001-03) detém os direitos materiais desta publicação. Os direitos referem-se à publicação do trabalho em qualquer parte do mundo, incluindo os direitos às renovaç̃ões, expansões e disseminações da contribuição, bem como outros direitos subsidiários. Todos os trabalhos publicados eletronicamente poderão posteriormente ser publicados em coletâneas impressas sob coordenação da Sustenere Publishing, da Companhia Brasileira de Produção Científica e seus parceiros autorizados. Os (as) autores (as) preservam os direitos autorais, mas não têm permissão para a publicação da contribuição em outro meio, impresso ou digital, em português ou em tradução. 